\title{
Las Políticas Ambientales de la Universidad de San Carlos de Guatemala: Aplicación en el Centro Universitario de Petén
}

\author{
The Environmental Policies of the University of San Carlos de \\ Guatemala: Application at the University Center of Petén
}

\section{Como citar el artículo}

Chán, J., (2019). Las Políticas Ambientales de la Universidad de San Carlos de Guatemala: Aplicación en el Centro Universitario de Petén. Revista Naturaleza, Sociedad y Ambiente, 6 (1), 15-28 DOI: https://doi.org/10.37533/cunsurori. v6i1.38

\section{José María Chán Mas}

Centro Universitario de Sur Oriente (CUNSURORI), Universidad de San Carlos de Guatemala.

Recibido: 29 de agosto de 2019 / Aceptado: 23 de octubre de 2019

Disponible en internet el 29 de noviembre de 2019

*Autor para correspondencia, correo electrónico: chemischan@gmail.com

\section{Resumen}

El artículo consiste en un análisis comparativo de las políticas ambientales de la Universidad de San Carlos de Guatemala y su aplicación en el Centro Universitario de Petén, este fue el objetivo principal que se planteó como interrogante de investigación, en donde se seleccionaron varias unidades de análisis para cada una de las políticas ambientales del área de docencia y de investigación. Los resultados de la investigación evidencias que las políticas ambientales no se han aplicado en el Centro Universitario de Petén, recién se ha elegido la comisión de ambiente, aun no se tiene ningún proyecto ambiental, no hay líneas de investigación concretas, en ningún momento se ha hecho un programa de diagnóstico ambiental, no todas las carreras cuentan con proyectos curriculares, el eje ambiental aún no se trabaja de manera inter y multidisciplinar. Por lo tanto, es necesario fortalecer la dimensión docente e investigación, para que en estas dos áreas se trabaje de manera transversal el tema ambiental. Las investigaciones encontradas en el registro universitario vienen de los estudiantes en proceso de graduación. No se identificaron investigaciones realizadas por docentes, publicaciones de artículos y/o ensayos académicos. El Centro Universitario de Petén, tiene una población de 3,029 estudiantes y 225 catedráticos, en el plan fin de semana se encuentra la mayor cantidad de estudiantes. Son estudiantes y profesores que participan en las treinta y un (31) carreras existentes, de las que trece (13) son de pregrado, catorce (14) de grado y cuatro (4) a nivel de maestría. Por lo que las actividades entrópicas y antrópicas generan un comportamiento ambiental en contra de la misma población universitaria.

Palabras clave: política ambiental, docencia, investigación, eje ambiental, cultura ambiental

\section{Abstract}

The article consists of a comparative analysis of the environmental policies of the University of San Carlos of Guatemala and its application in the University Center of Petén, this was the main objective that was raised as a research question, where several units of analysis were selected. for each of the environmental policies of the teaching and research area. The results of the research evidence that environmental policies have not been applied in the University Center of Petén, the environment committee has just been chosen, there is still no environmental project, there are no specific research lines, at no time has made an environmental diagnostic program, not all careers have curricular projects, the environmental axis is not yet worked inter and multidisciplinary.Therefore, it is necessary to strengthen the teaching and research dimension, so that in these two areas the environmental issue is dealt with transversally. The research found in the university registry comes from students in the process of graduation. We did not identify research carried out by teachers, articles and / or academic essays. The Petén University Center, has a population of 3,029 students and 225 professors, in the weekend plan is the largest number of students. They are students and professors who participate in the thirty-one (31) existing careers, of which thirteen (13) are undergraduate, fourteen (14) of degree and four (4) at the master's level. Therefore, entropic and anthropic activities generate an environmental behavior against the same university population.

Keywords: environmental policy, teaching, research, environmental axis, environmental culture 


\section{Introducción}

El presente artículo es un trabajo de investigación producto de la inquietante tarea de conocer más acerca del tema ambiental. Este estudio toma las políticas ambientales de la Universidad de San Carlos de Guatemala y analiza su aplicación en el Centro Universitario de Petén.

Este estudio es importante debido a que en el Centro Universitario de Petén no se han tomado en serio las políticas ambientales surgidas en el año dos mil catorce (2014), por lo que no se cuenta con un programa ambiental definido, y las líneas de investigación no están estipuladas.

Es necesario considerar que muchas universidades ya están trabajando para convertirse en universidades verdes, universidades con sistemas de gestión ambiental, orientadas a estar haciendo investigación constantemente y a que sus docentes hagan publicaciones de investigaciones propias.

Hay muchas declaraciones que defienden el papel de la universidad a nivel socioambiental, una de esas declaraciones es, La Declaración de Piura (2012), afirmando que cada universidad debe adoptar políticas de responsabilidad socioambiental dentro de sus fundamentos estratégicos y en el marco de la Política Nacional del Ambiente, contextualizado y personalizado por cada universidad.

Para (Rodríguez y Espinoza 2017), "las políticas son el conjunto de objetivos, principios, criterios y orientaciones generales para la protección del medio ambiente de una sociedad particular". Por tal motivo y para la obtención de los resultados se utilizó como camino para la investigación la selección de una unidad de análisis para cada política ambiental en las áreas de docencia e in- vestigación procediendo al análisis comparativo y constatar la aplicación de la política en esas áreas de conocimiento, esto se hizo a través de la elaboración de una tabla en donde se describen los aspectos del estudio.

A través de lo expuesto anteriormente se llegó al resultado de la indagación, estos demuestran que las políticas ambientales no son conocidas en el centro universitario de Petén, que aún no tienen aplicabilidad debido a que no cuentan con un proyecto ambiental, líneas de investigación y un diagnóstico ambiental, que refleje la crisis ambiental, esta información obtenida se detalla con amplitud al final del documento.

\section{Planteamiento del Problema}

El Centro Universitario de Petén, según organigrama general de la Universidad de San Carlos de Guatemala, es uno de los centros regionales de esta casa de estudios. El CUDEP se ubica en el área central en el parque las Estelas, Santa Elena, Flores, Petén, creado en el año de 1987, e iniciando actividad académica en el año 1988. Posteriormente se incorporan las carreras del plan fin de semana, surgiendo en el año 1995 la carrera de Profesorado de Enseñanza Media en Pedagogía y en 1998 la Licenciatura en Educación Ambiental. Esta última fecha es importante para fines de la investigación, ya que la Educación Ambiental EA constituye un pilar fundamental en la formación de los ciudadanos y en brindar mejores condiciones al Centro Universitario, para que estas sean más adecuadas para todos, debido al cuidado del medio ambiente.

Es importante considerar también que la actividad antrópica genera consecuencias, y eso tiene que ver con aspectos como la población estudiantil, para conocer este contexto se proporcionan algunos datos. 
Según registro de inscripciones 2018, el Centro Universitario de Petén, tiene una población de 3,029 estudiantes. Según responsables de tesorería, existen 75 catedráticos titulares, 150 por contrato, solamente en las carreras del plan fin de semana hay 60 docentes y 15 administrativos. Según catálogo de carreras, Cudep, 2018, las carreras existentes a nivel de pregrado son 13 , a nivel de grado 14 y a nivel de maestría 4 , en total son 31 .

Las carreras del plan fin de semana del centro universitario de Petén, constituyen la mayor parte de la población, solamente las carreras en Educación reportan 559 estudiantes, aglutinando más población que todas las demás carreras. Este fenómeno se debe a que muchos jóvenes trabajan durante la semana teniendo opción de continuar estudios solamente los sábados. El centro recibe jóvenes de distintos contextos sociales, estilos de vida, hábitos culturales muy diversos, personas de áreas rurales que viajan al área central, etc. Todas estas realidades se confrontan en un mismo tema, el comportamiento ambiental, (Hübner, 1923) define las costumbres como "la reiteración de un mismo acto acompañada de la convicción de responder a una necesidad jurídica".

A través de la observación directa se perciben algunos problemas ambientales dentro de las instalaciones de la universidad, tales como; casos de abundante basura en los pasillos y aulas de estudio, desperdicio de agua en los servicios sanitarios, consumo de energía eléctrica excesiva, desperdicio de papel, canales de desagües en condiciones desfavorables.

Estudio realizado en el dos mil quince en las carreras de educación, trabajo social y ciencias de la comunicación, refleja que para la opinión de los catedráticos existen serios problemas ambientales de todo tipo en la universidad.

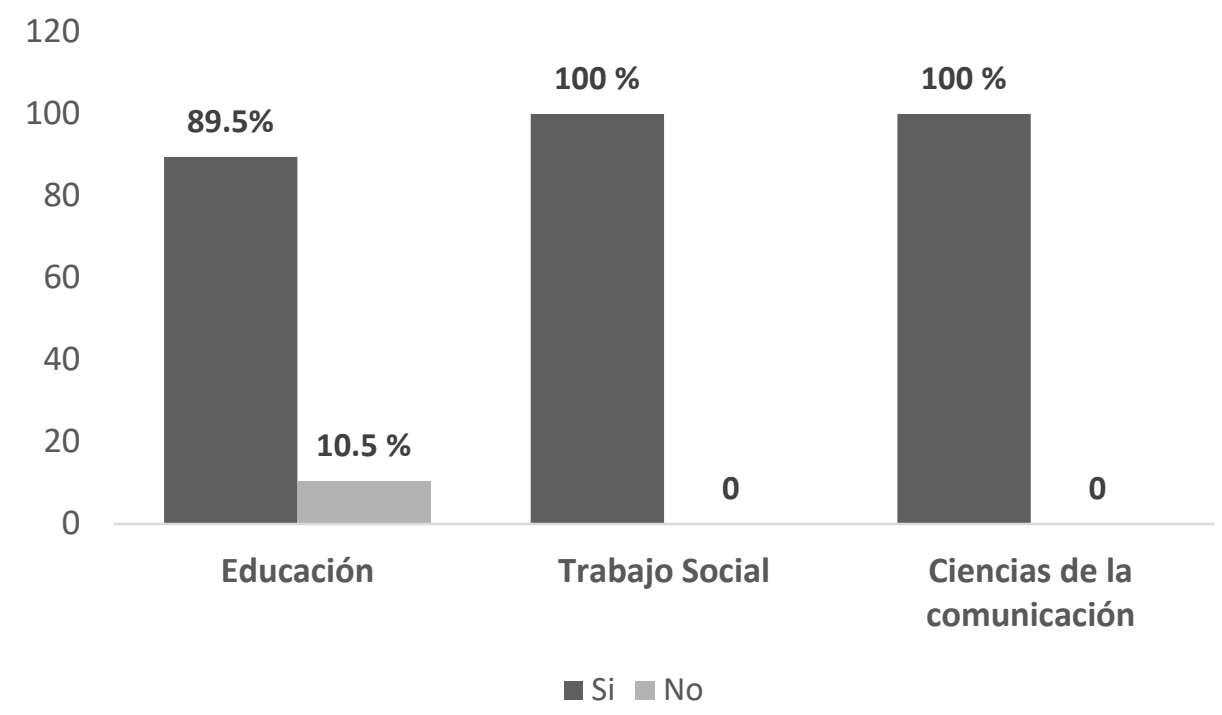

Figura No. 1

Fuente: (Chán, 2015), tesis de maestría, análisis de la presencia del eje ambiental en las carreras de Educación, Trabajo Social y Ciencia de la Comunicación del Centro Universitario de Petén. p.62 
Esta realidad puede ser transformada con la aplicación de las políticas ambientales, según Acta 13-2014 del Consejo Superior Universitario, "cada política tiene su correspondiente plan o planes estratégicos con sus respectivos programas. En cada programa se plantean las ideas de proyectos que deben ser desarrolladas por las unidades responsables". Estas políticas surgieron como una propuesta presentada por la Comisión Ambiental Permanente del Consejo Superior Universitario de la Universidad de San Carlos de Guatemala, cuyo objetivo general literalmente indica; construir en la comunidad universitaria una cultura ambiental sostenible, por medio de estrategias coherentes, programas y proyectos integrados e integrales de fortalecimiento del desarrollo sostenible en las áreas de investigación, docencia, extensión y administración, con el fin de conservar y mejorar las condiciones ambientales en los espacios universitarios, desarrollando campus ambien- talmente sanos y seguros para una comunidad comprometida con el ambiente. (Política Ambiental USAC, 2014).

Enmarcados en este aspecto, surge la pregunta de investigación planteada de la siguiente manera: ¿El Centro Universitario de Petén, aplica las políticas ambientales de la Universidad de San Carlos de Guatemala?

\section{Metodología}

Para el desarrollo de la investigación se verifica la aplicación de las políticas ambientales de la Universidad de San Carlos de Guatemala en el Centro Universitario de Petén, identificando estas políticas ambientales, en las áreas de docencia e investigación, comparando y analizando lo establecido para cada política con una unidad de análisis, tal como se presenta en la siguiente tabla.

\begin{tabular}{|c|c|c|c|c|}
\hline No. & Área & Políticas ambientales & $\begin{array}{l}\text { Lo establecido para cada } \\
\text { política }\end{array}$ & Unidad de análisis \\
\hline 1. & Docencia & $\begin{array}{l}\text { Enfoque ambiental en las } \\
\text { unidades académicas. }\end{array}$ & $\begin{array}{l}\text { Incorporación del tema } \\
\text { ambiental. }\end{array}$ & $\begin{array}{l}\text { Pensum de estudio } \\
\text { de las carreras }\end{array}$ \\
\hline \multirow{5}{*}{2.} & \multirow{5}{*}{ Investigación } & $\begin{array}{l}\text { Sistema de actualización } \\
\text { curricular. }\end{array}$ & $\begin{array}{l}\text { Readecuación curricular. } \\
\text { Eje transversal en los } \\
\text { perfiles y planes de } \\
\text { estudio, en todas las } \\
\text { unidades académicas. }\end{array}$ & Proyecto curricular \\
\hline & & $\begin{array}{l}\text { Sistema de educación } \\
\text { ambiental superior. }\end{array}$ & Maestrías en ambiente. & \\
\hline & & $\begin{array}{l}\text { Proyectos interdisciplinar } \\
\text { y multidisciplinar. }\end{array}$ & $\begin{array}{l}\text { Programa de elaboración } \\
\text { de diagnóstico ambiental. }\end{array}$ & \\
\hline & & Eje transversal. & $\begin{array}{l}\text { Tesis de grado y } \\
\text { postgrado. }\end{array}$ & $\begin{array}{l}\text { Coordinación } \\
\text { académica. }\end{array}$ \\
\hline & & & & $\begin{array}{l}\text { Área de } \\
\text { investigación }\end{array}$ \\
\hline
\end{tabular}

Tabla 1. Políticas ambientales y su unidad de análisis 
Se verificará la aplicación de las políticas ambientales en el Centro Universitario de Petén, en las áreas de docencia e investigación, asignando una unidad de análisis con la que se pretende encontrar los elementos establecidos en las Políticas Ambientales de la USAC, estas unidades de análisis son, por ejemplo, los proyectos curriculares (si la carrera cuenta con ello), sus planes y/o pensum, investigaciones a nivel de grado y postgrado. Entrevista a coordinación académica, coordinadores de carrera, encargado de investigación, responsable de postgrado y director del centro universitario.

Posteriormente se hace la discusión y análisis de los resultados obtenidos, describiendo y redactando las conclusiones de la investigación.

\section{Desarrollo teórico}

\section{La Política}

"Es la actividad en la que el ser humano se realiza" (Arendt, 2009) estas actividades generan políticas de todo tipo, las cuales cuando son establecidas a partir de realidades y necesidades concretas, coadyuvan al bienestar común.

La acción única actividad que se da entre los hombres sin la mediación de cosas o materia, corresponde a la condición humana de la pluralidad, al hecho de que los hombres, no el Hombre, vivan en la Tierra y habiten en el mundo. (Arendt, 2009).

\section{El poder hacer}

Esto tienes que ver con el poder de hacer transformaciones concretas es decir "el poder es parte de nuestro accionar" (Foucault, 1971). El poder refleja las formas en que los seres humanos ejercer gobernanza sobre otros, influyendo estos, positiva o negativamente. Cuan- do se influye positivamente como por ejemplo en la transformación del medio ambiente, el poder de decisión, de obrar e inversión genera un bienestar común fuera de los intereses particulares, individualistas y destructores.

La acción es la actividad política que el ser humano realiza, estas, a veces van en favor del medio ambiente y otras veces en contra, las cuales reciben el nombre de actividades antrópicas del ser humano. Las políticas ambientales cobran vigencia cuando las autoridades universitarias promueven su aplicación. Utilizan el poder para el bien común.

\section{Las políticas ambientales}

Según (ISO 14001, 2015), las políticas ambientes: La política ambiental debe ser definida al más alto nivel directivo, el cual tiene que poseer la capacidad y las atribuciones necesarias para poder modificar y dirigir el Sistema de Gestión Ambiental, ya que la política es la que impulsa la implementación y mejora del Sistema de Gestión Ambiental.

La política ambiental debe ser una declaración breve, pública y documentada en la que la empresa pone sus intenciones para con el medio ambiente, los objetivos generales y los principios que guiará la mejora continua. Debe ir firmada por la alta dirección, ya que es un documento escrito donde se exponen todos los compromisos que persigue la organización con respecto al medio ambiente. Deberá ser compresiva y detallada, realista y evitando objetivos que de antemano se sabe que son inalcanzables. 
Políticas ambientales de la Universidad de San Carlos de Guatemala

Las políticas ambientales (2014), literalmente dicen:

\section{Área de Docencia}

a) Institucionalizar el enfoque ambiental en las unidades académicas.

b) Fortalecer el sistema de actualización curricular universitario, orientándolo hacia el desarrollo sostenible, con el objeto de que todos los egresados de la USAC cuenten con competencias y principios de responsabilidad en la sostenibilidad ambiental, de gestión de riesgo ante la vulnerabilidad del país y de adaptación al cambio climático y mitigación de sus efectos.

c) Crear el Sistema de Educación Ambiental Superior en la Universidad de San Carlos de Guatemala.

\section{Área de Investigación}

a) Incentivar proyectos de investigación, interdisciplinaria y multidisciplinaria, buscando integrar a las unidades académicas, sobre manejo ambiental, gestión de riesgo ante la vulnerabilidad del país y para la adaptación al cambio climático y mitigación de sus efectos.

b) Incorporar el componente ambiental, gestión de riesgo, recursos naturales, diversidad biológica y cultural, adaptación al cambio climático y mitigación de sus efectos, como eje transversal, en las investigaciones a todo nivel, incluyendo las tesis de grado y posgrado, así como la investigación básica y aplicada de las unidades académicas.

Según (Rodríguez y Espinoza 2017), indican que "las políticas son el conjunto de objetivos, principios, criterios y orientaciones generales para la protección del medio ambiente de una sociedad particular. Esas políticas se ponen en marcha mediante una amplia variedad de instrumentos y planes".

\section{Las políticas ambientales en la universidad}

La Declaración de Kyoto (Asociación Internacional de Universidades, 1993), señala que: La necesidad de cooperación con otros sectores de la sociedad para el desarrollo de medidas prácticas y políticas, anima a las universidades a revisar sus propias operaciones y a desarrollar e implementar un plan de acción propio para la consecución de los objetivos de la declaración.

La Declaración de Piura (2012), reafirma "la importancia que en cada universidad se adopte políticas de responsabilidad socioambiental dentro de sus fundamentos estratégicos y en el marco de la Política Nacional del Ambiente, el cual podrá ser contextualizado y personalizado por cada universidad". Las políticas ambientales se visualizan como un conjunto de normas cuya finalidad consiste en proteger el medio ambiente. Reducir las actividades de impacto ambiental. Existen modelos para la gestión ambiental, tal como las normas ISO 14001, considerando acciones concretas tales como: planificar, hacer, verificar y actuar. Las universidades Certificadas con ISO 14001, están consideras universidades que aplican la gestión ambiental por lo tanto protegen el medio ambiente.

Muchas universidades del mundo están trabajando para obtener su certificación ambiental, o certificación de las normas ISO, esta certificación la han logrado aquellos que tienen un sistema de gestión ambiental. Para ello es indispensable conocer que "La Educación Ambiental forma parte integrante del proceso educativo, debería girar en torno a problemas concretos y tener carácter inter- 
disciplinario". (Conferencia de Tblisi, 1977).

Según aprobación del Consejo Superior Universitario en sesión celebrada el 30 de julio de 2014 Punto Sexto, Inciso 6.2 Acta 13-2014, se aprueban las políticas ambientales de la USAC, para ser aplicadas en cada uno de los Centro Universitarios.

\section{La investigación ambiental en el CUDEP}

Del área de investigación se derivan los aportes científicos para mejorar los sistemas actuales del Centro Universitario, para ello es necesario contar con líneas de investigación acordes a las necesidades y demandas. Contar con programas de investigación, estudios que permitan dar soluciones, para no reducir la investigación únicamente a los trabajos de graduación que realizan los egresados, para que estos documentos no terminen en los archivos y biblioteca de la universidad.

Desde el enfoque de las políticas ambientales se debe considerar la investigación como un proyecto interdisciplinar y multidisciplinar que integre la unidad académica. Debe incorporar el componente ambiental y todos los derivados de este, para desarrollarlos a través de un eje transversal, que incluya las tesis de grado y posgrado.

El reporte de las investigaciones realizadas en el CUDEP en los últimos cuatro años, indica que estas han sido realizadas por estudiantes en la fase de graduación, por otra parte, no existen aportes de docentes realizando y promoviendo investigación científica, tampoco de publicaciones, dígase artículos $\mathrm{o}$ ensayos académicos.
Es necesario estimular y aprovechar la investigación de los estudiantes, incorporando estos estudios a programas organizados de investigación. Que ella no sea vista como algo propio de los estudiantes, sino en conjunto, todo el Centro debe hacer investigación, todo el centro debe comprometerse con las soluciones de los problemas socioambientales.

\section{EI Sello Verde}

Se entiende como la compra verde, compra o contratación de obras, bienes, servicios o suministros, que incluyen criterios de sostenibilidad en su producción y distribución. (Políticas Ambientales, USAC 2014).

Según (Wille, Wunderlich y Barrios, s.f), indican que: Un sello verde es una etiqueta que brinda información sobre el impacto ambiental de producir, cultivar, cosechar, procesar, transportar o usar un producto. Proporciona información al consumidor y lo ayuda a escoger los productos que consume. Los sellos son otorgados por grupos independientes, sin fines de lucro.

\section{Realidad socio-ambiental}

Con tantas preocupaciones sobre el ambiente es decisivo entender lo que está sucediendo realmente en el país, si las legislaciones realmente están provocando cambios trascendentales, si los gobernantes tienen una seria y comprometida responsabilidad con el medio ambiente.

Por el contrario, cada vez son más los índices de contaminación en el país, en el año 2016 se hizo un estudio dirigido por la Organización Mundial de la Salud, reportando que la ciudad capital de Guatemala, tiene 41 microgramos de contaminación del aire. La OMS atribuye a 
la contaminación más de 7 millones de muertes, causadas por la elevada concentración de partículas pequeñas y finas que provocan diversas enfermedades cáncer de pulmón $y$ enfermedades respiratorias- $y$ aumenta el riesgo de derrame cerebral y cardiopatía.

Según (Monterroso, 2010) en el Perfil Ambiental de Guatemala, expresa: Los procesos de dominación y dependencia, de integración y de democracia, desarrollados en Guatemala a partir de la apertura democrática de 1986, han contribuido más al agotamiento y deterioro de los recursos naturales y culturales, y a la destrucción ambiental, que al desarrollo sostenible del país.

En primer lugar, las políticas ambientales que se han generado a nivel mundial no son el producto de una decisión tomada por los representantes del sistema económico que se impulsa, por su deseo de contribuir al cuidado y conservación de los recursos naturales y culturales del planeta. Son, más bien, el resultado de la presión ejercida por el movimiento ecologista internacional, al cual pertenecen quienes sí tienen preocupación por el planeta.

En segundo lugar, cuando ese tipo de políticas se expresa a nivel nacional, no es porque con ellas se busque detener el creciente deterioro ambiental; se trata, más bien, de instrumentos para salvaguardar el sistema productivo en el que se basa el crecimiento económico.

Es urgente que la preocupación por el medio ambiente se refleje en cambios concretos, que todos los sectores de la población se involucren, que la universidad cumpla su función social, atacando, contrarrestando, investigando y proponiendo soluciones alternas a la gran crisis ambiental existente. Que no haya intenciones oscuras con pretensión de sobreguardar un sistema productivo de tipo económico.

\section{La cultura ambiental sostenible}

Es importante desarrollar una cultura ambiental que se refleje en los patrones y estilos de vida de la comunidad universitaria, que los salones de clase no queden llenos de basura después de haber servido de aula del saber, que los espacios y corredores se mantengan libres de basura, que el uso del agua y la electricidad sea de forma racional. La cultura ambiental es aquel modo de vida que no daña los sistemas naturales, preserva la vida, desarrolla actividades a favor del medio ambiente.

Según (García y Vega, 2009), afirma que: La cultura interpreta un papel clave en la definición y puesta en marcha de políticas de sostenibilidad. Como mediadora entre la conducta y el ambiente, forma la estructura a través de la que la interacción entre las personas y sus entornos cambiantes facilita o limita el desarrollo sostenible. En este proceso, la educación ambiental y la transmisión de valores constituyen herramientas básicas imprescindibles para conducir el cambio social y afrontar los retos del cambio climático.

\section{La racionalidad ambiental}

La racionalidad ambiental es entendida como el proceso a través del cual el ser humano reflexiona sobre sus actividades antrópicas y sobre su entropía con el planeta tierra. Trata de buscar otras opciones de vida que a él y a su prójimo le permitan continuar con el ciclo de la civilización humana.

Según (Leff, 2004), indica que: La racionalidad ambiental construye nuevos mundos de vida en la rearticulación entre la cultura y la naturaleza que, más allá de una voluntad de forzar 
la identidad entre lo real y lo simbólico en un monismo ontológico, reconoce su dualidad y diferencia en la constitución de lo humano. Del desquiciamiento de la naturaleza y de la razón que se expresa en la crisis ambiental, emerge una nueva racionalidad para reconstruir el mundo, más allá de la ontología y la epistemología, desde la otredad y la diferencia.

\section{La institucionalidad de las políticas ambi- entales}

Las políticas ambientales de la USAC, piden que el enfoque ambiental se institucionalice en las unidades académicas, pero qué es esto, cómo se debe institucionalizar. Según (Rodríguez 2014) "La política se institucionaliza allí y cuando se organiza, se articula y se radica en instituciones".

\section{Resultados}

De las 31 carreras que existen en el Centro Universitario de Petén, a nivel de pregrado dos (2) tienen explicito el nombre ambiental, a nivel de grado dos (2) y a nivel de postgrado dos (2), en total seis tienen enfoque ambiental. Estas son, Profesorado de Enseñanza Media en Pedagogía con Orientación en Medio Ambiente, Técnico en Conservación y Manejo de Bosques Tropicales, Licenciatura en Educación Ambiental, Ingeniería Forestal, Maestría en Educación con Orientación en Medio Ambiente y Maestría en Educación con Ambientalización Curricular.

De las demás carreras algunas cuentan con uno o dos cursos ambientales, otras como trabajo social, derecho, medicina, profesorado en matemática y física, pedagogía, no tienen incorporado cursos ambientales en su pensum de estudios. En el Área de Docencia, el modelo de la tabla 2, permitió constatar que el enfoque ambiental no está institucionalizado en la unidad académica, debido a que aún no hay personal directamente comprometido con el fortalecimiento del sistema de actualización curricular universitario y de orientarlo hacia el desarrollo sostenible.

En la mayoría de los diseños curriculares de las carreras existentes, en sus perfiles de egreso no presentan competencias y principios de responsabilidad en la sostenibilidad ambiental, de gestión de riesgo ante la vulnerabilidad del país y de adaptación al cambio climático y mitigación de sus efectos.

El Centro Universitario de Petén no cuenta con un Sistema de Educación Ambiental a nivel general, existe una carrera en Educación Ambiental que hace el esfuerzo por contribuir con el medio ambiente pero que aún no ha trascendido a todo el Centro, esto se afirma porque aún no hay un sistema que maneje responsablemente el tema ambiental a nivel de unidad académica, se hacen algunas cosas aisladamente en algunas carreras, tales como rotulaciones, reciclaje, siembra de arbolitos, pero no es un trabajo de conjunto.

En el Área de Investigación, el modelo de la tabla 3, permitió constatar que en cuanto a estas políticas se realizan proyectos de investigación con criterios ambientales en algunas carreras de pregrado, grado y postgrado, se hacen intentos para incentivar proyectos de investigación, interdisciplinaria y multidisciplinaria, pero estos aun no forman parte de la integración total del centro.

Los temas de investigación son elegidos a criterio de los estudiantes, en los casos de las carreras con orientación ambiental, la tendencia de investigación va por esta misma línea. La realidad es que estas investigaciones 
quedan archivadas en coordinación académica y en la biblioteca, y no se aprovechan sus resultados para provocar cambios transcendentales, los estudiantes realizan estas investigaciones como proceso de graduación.

Para incorporar el componente ambiental en esta realidad universitaria quedan pendientes tareas tales como; gestión de riesgo, conservación de la diversidad biológica y cultural, adaptación al cambio climático y mitigación de sus efectos. Recientemente el Consejo Directivo conformó una comisión de ambiente, para velar por estos temas, pero es un trabajo que aún empieza a coordinarse. El tema ambiental no cumple las funciones de eje transversal, en los procesos de investigación no se tiene una línea de investigación y/o programas de investigación, tampoco programas de encuentro multidisciplinar que se incluyan en las tesis de grado y posgrado.

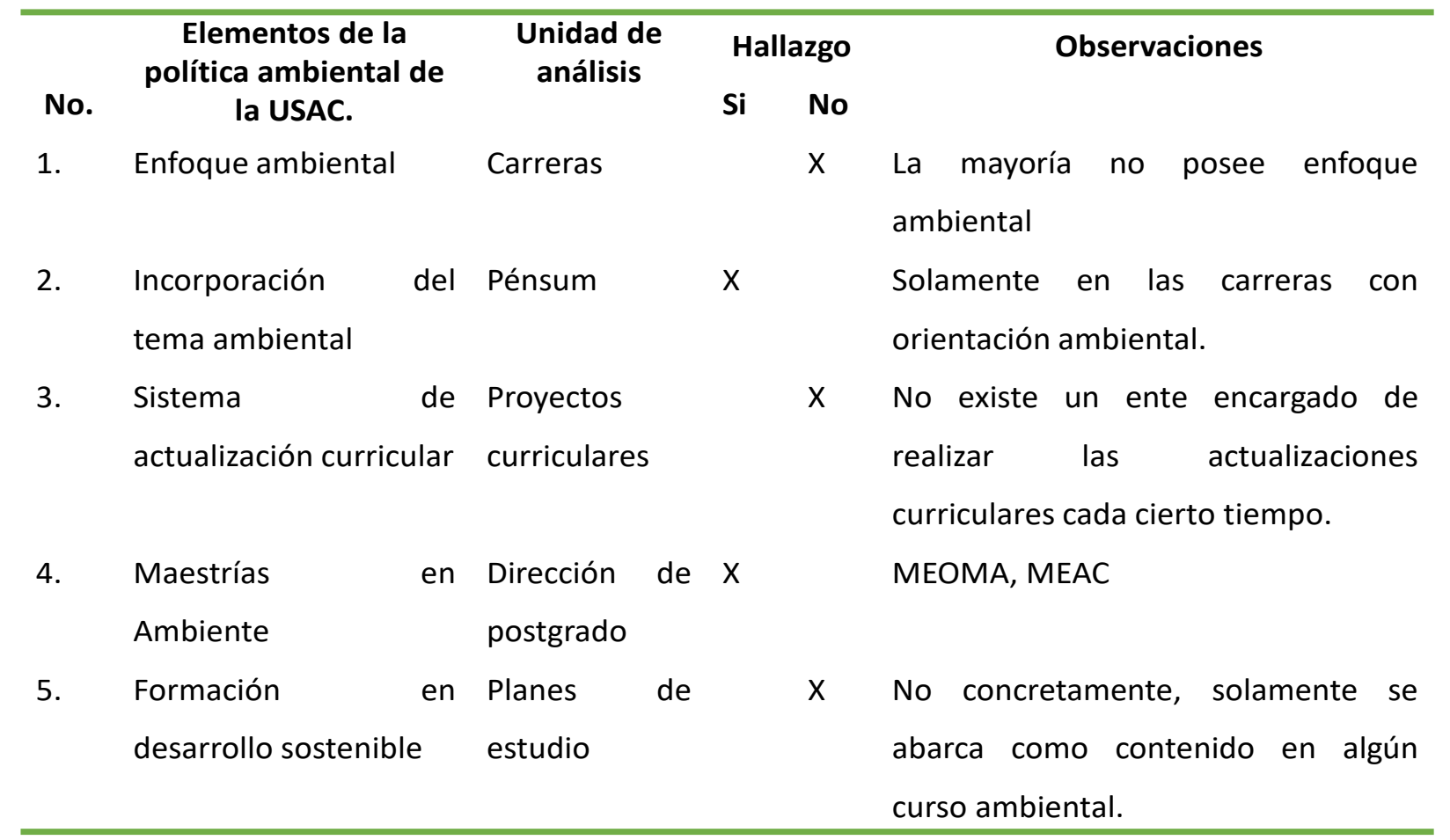

Tabla 2. Elementos de las políticas ambientales en el área de docencia. 


\begin{tabular}{|c|c|c|c|c|c|}
\hline \multirow[b]{2}{*}{ No. } & \multirow{2}{*}{$\begin{array}{l}\text { Elementos de la } \\
\text { política ambiental } \\
\text { de la USAC. }\end{array}$} & \multirow[b]{2}{*}{$\begin{array}{l}\text { Unidad de } \\
\text { análisis }\end{array}$} & \multicolumn{2}{|c|}{ Hallazgo } & \multirow[b]{2}{*}{ Observaciones } \\
\hline & & & Si & No & \\
\hline 1. & $\begin{array}{l}\text { Proyecto } \\
\text { interdisciplinar y } \\
\text { multidisciplinar }\end{array}$ & $\begin{array}{l}\text { Unidad } \\
\text { académica }\end{array}$ & & $x$ & $\begin{array}{l}\text { No existen proyectos en el Cudep } \\
\text { de esta índole. }\end{array}$ \\
\hline 2. & $\begin{array}{l}\text { Programa de } \\
\text { elaboración de } \\
\text { diagnóstico } \\
\text { ambiental }\end{array}$ & $\begin{array}{l}\text { Coordinación } \\
\text { académica }\end{array}$ & & $x$ & $\begin{array}{l}\text { Aún no existe ningún programa de } \\
\text { diagnóstico ambiental en el Cudep. }\end{array}$ \\
\hline 3. & $\begin{array}{l}\text { Eje ambiental en las } \\
\text { tesis de grado y } \\
\text { postgrado }\end{array}$ & $\begin{array}{c}\text { Área de } \\
\text { investigación }\end{array}$ & $x$ & & $\begin{array}{l}\text { En las carreras con orientación } \\
\text { ambiental exclusivamente. }\end{array}$ \\
\hline 4. & $\begin{array}{l}\text { Línea de } \\
\text { investigación }\end{array}$ & $\begin{array}{c}\text { Área de } \\
\text { investigación }\end{array}$ & & $x$ & $\begin{array}{l}\text { El tema ambiental, no se tiene } \\
\text { establecido como una línea de } \\
\text { investigación. }\end{array}$ \\
\hline 5. & $\begin{array}{l}\text { Programas de } \\
\text { investigación }\end{array}$ & $\begin{array}{c}\text { Área de } \\
\text { investigación }\end{array}$ & & $x$ & Con enfoque ambiental no existe. \\
\hline 6. & $\begin{array}{l}\text { Programas de } \\
\text { encuentro }\end{array}$ & Postgrado & & $x$ & $\begin{array}{l}\text { A nivel de centro universitario no } \\
\text { se implementa. }\end{array}$ \\
\hline
\end{tabular}

Tabla 3. Elementos de las políticas ambientales en el área de investigación.

\section{Análisis y discusión}

Este análisis de los resultados, surte efecto a partir de la interrogante $¿$ El Centro Universitario de Petén, aplica las políticas ambientales de la Universidad de San Carlos de Guatemala?

En la metodología se definió el proceso de análisis que se utilizaría para recabar la información necesaria para responder a la pregunta de investigación. Este camino utilizado consistió en tomar las políticas ambientales de las áreas de docencia e investigación y compararla con una unidad de análisis previamente seleccionada. Los resultados reflejan que aún hay un gran trabajo por hacer en el Centro Universitario de Petén, y que es un reto para la comisión de ambiente nombra por el consejo directivo en el 2018, lograr que las políticas ambientales y sus objetivos se cumplan.
El Centro Universitario de Petén, es una casa de estudio que forma profesionales en treinta y una áreas de conocimiento diversos, estas personas que egresan de la universidad van al campo laboran en donde multiplican lo aprendido. Es por eso necesaria la transversalidad ambiental, para que se coadyuve a promover una cultura ambiental en los contextos de estudio y laboral.

EI CUDEP, con sus carreras ambientales debe impregnar a todas las demás de ese componente, haciendo una labor, interdisciplinar, multidisciplinar y transdisciplinar.

El centro universitario de Petén debe cumplir con los lineamientos y acciones que exige la política ambiental de la USAC, en las áreas de docencia e investigación para desarrollar una cultura ambiental en las carreras que se imparten en la universidad. 
El problema principal que se identifican en la investigación es la falta de aplicación de las políticas ambientales de la Universidad de San Carlos de Guatemala en el Centro Universitario de Petén, lo cual repercute en la necesidad de:

a. Crear una cultura ambiental sostenible

b. Una interdisciplinariedad, multidisciplinariedad y transversalidad ambiental

c. Un sistema de actualización curricular

d. Un sistema de educación ambiental superior

e. Formación en desarrollo sostenible

f. Programa de elaboración de diagnóstico ambiental

g. Línea de investigación ambiental

h. Programas de investigación ambiental

i. Programas de encuentro ambiental interdisciplinar.

Son treinta y una carreras que existen en el CUDEP, en donde para que los proyectos de investigación interdisciplinaria y multidisciplinaria sean incorporados, es necesario que: la complejidad de los problemas ambientales sea resueltos utilizando nuevas estrategias que superen la investigación disciplinar, que se conjuguen aspectos de análisis desde la mirada propia de cada una, para enriquecer el objeto de estudio. Un proyecto de investigación multidisciplinar que se encargue de dividir los objetivos entre las disciplinas de cada uno de los investigadores y el producto final sea una recopilación disciplinar de cada visión.

Por tanto, en cuanto al objetivo general de las políticas ambientales, "construir en la comunidad universitaria una cultura ambiental sostenible, por medio de estrategias coherentes, programas y proyectos integrados $\mathrm{e}$ integrales de fortalecimiento del desarrollo sostenible..." será una tarea que la comisión de ambiente recién nombrada por el consejo directivo, tendrá que trabajar para lograr una cultura ambiental sostenible, con la participación de todos los actores universitarios.

Desde la educación ambiental es necesario que se conserven y mejoren las condiciones ambientales en los espacios del centro universitario de Petén, obteniendo ambientes sanos y seguros para la comunidad universitaria.

Las tesis que se han hecho a partir del año 2014 a la fecha, son egresados de la carrera de educación ambiental y cuatro proveniente de maestría. Eso significa que las demás carreras no le están apuntando a investigar sobre temas ambientales. Estas investigaciones se están enfocando más en temas tales como: desechos sólidos, contaminación ambiental, educación ambiental, flora y fauna, aguas servidas, cambio climático, concesión forestal.

El reporte de las investigaciones realizadas en el CUDEP en estos últimos cuatro años, los estudios han sido realizadas por estudiantes en la fase de graduación, es necesario y urgente que también los docentes promuevan y realicen investigación científica, que escriban artículos y/o ensayos académicos.

\section{Conclusiones}

En el Centro Universitario de Petén hace falta un proyecto en donde se incluyan las políticas ambientales de la Universidad de San Carlos de Guatemala, con programas, planes y líneas de investigación específicos, que estos sean integrales y de fortalecimiento para el desarrollo sostenible. 
Conservary mejorarlas condiciones ambientales en los espacios universitarios, desarrollando ambientes sanos y seguros para la comunidad universitaria, es una tarea impostergable.

Fortalecer el área de docencia e investigación es urgen para la unidad académica de Petén.

Las políticas ambientales de la Universidad de San Carlos de Guatemala están vigentes desde el año dos mil catorce, por lo que hasta la fecha no han sido aplicadas en el CUDEP.

Las investigaciones ambientales que se realizan en el centro universitario están enfocadas en la línea de los trabajos de graduación en las carreras de educación ambiental y maestría con orientación ambiental, no hay registro de docentes que estén realizando investigación científica para el desarrollo de la academia universitaria.

No existen programas de encuentro en donde se aborden los temas ambientales con seriedad y con prontitud.

Hace falta que se elabore un programa de diagnóstico ambiental que evidencie las necesidades y problemas ambientales más urgentes de la universidad.

\section{Recomendaciones}

Qué la comisión ambiental recientemente nombrada en el CUDEP, trabaje para la aplicación de las políticas ambientales, considerando las áreas de docencia e investigación, así las otras que comprende.

Que las autoridades del centro tomen medidas concretas con relación al proceder de la universidad, es decir que se refleje una verdadera cultura ambiental en todos los actores.
Qué se elabore en el Centro Universitario de Petén un programa de diagnóstico ambiental para detectar los problemas originados de las actividades antrópicas.

Qué las autoridades hagan todo el esfuerzo por mantener un centro seguro, higiénico y saludable, hacia la consecución de una universidad verde.

Que se realicen actividades de encuentro formativo y de reflexión e investigación, para exponer temas serios que atañen a la educación superior.

\section{Referencias}

Arendt, Hannah (2009) La condición humana. PAIDÓS. Buenos Aires. Barcelona. México. p.21

Catálogo de Carreras, (2018) Universidad de San Carlos de Guatemala, Centro Universitario de Petén, Departamento de Control Académico.

Chán Mas, José María, (2015). Análisis de la presencia del eje ambiental en las carreras de educación, trabajo social y ciencias de la comunicación. Tesis. Centro Universitario de Petén. p.62

Chomsky N., Foucault M. [Milito Asno]. (2017, junio 11). El Poder. [Archivo de video]. Recuperado de https://www.youtube. com/watch?v=V6CiCbBxg7c\&t=174s

Conferencia Intergubernamental sobre Educación Ambiental. (1977). Tbilisi (URSS). Unesco y cooperación del PNUMA. p. 20

Declaración de Kyoto (1993) Desarrollo Sostenible. Asociación Internacional de Universidades.

Declaración de Piura (2012), Universidad, Gestión Ambiental y Desarrollo Sostenible. 
García Mira, R., y Vega Marcote, Pedro (2009). Sostenibilidad, valores y cultura ambiental. Revista. Ediciones Pirámide. Recuperado de https://www.edicionespiramide.es/libro.php?id=2317131

Ginebra (12 de mayo de 2016). Guatemala figura entre las ciudades más contaminadas. Prensa Libre.

Google Maps (2018) Universidad de San Carlos de Guatemala.
Húbner Gallo, Agustín Squella. (1923). Introducción al derecho. p.141.

Leff Enrique, (2004). Racionalidad Ambiental: La reapropiación social de la naturaleza. Siglo XXI editores, s.a. de c.v. prólogo.

Monterroso, Neptalí. (2010-2012). El Contexto Político como condicionante de la gestión ambiental. Perfil Ambiental de Guatemala. Universidad Rafael Landívar. Instituto de Agricultura, Recursos Naturales y Ambiente. p.18.30

\section{Sobre autor}

\section{José María Chán Mas}

Guatemalteco, Licenciado en Pedagogía y Ciencias de la Educación, Maestro en Educación y Ambientalización Curricular, estudiante del sexto semestre de doctorado en investigación para el desarrollo social, Cunsurori. Revisor de tesis en el Centro Universitario de Petén durante el año 2017, Coordinador de la Maestría en Docencia Universitaria del Centro Universitario de Petén 2017-2018. Ejerce actividades docentes en el Ministerio de Educación 2005-2020.

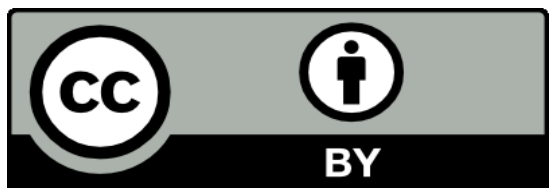

Este texto está protegido por una licencia CreativeCommons 4.0.

Esta licencia permite que otros distribuyan, mezclen, adapten y desarrollen su trabajo, incluso comercialmente, siempre y cuando le den crédito por la creación original. 